Pure Appl. Chem., Vol. 73, No. 8, pp. 1257-1260, 2001.

(C) 2001 IUPAC

\title{
Teaching green chemistry. Third-year-level module and beyond*
}

\author{
Colin L. Raston ${ }^{\dagger}$ and Janet L. Scott \\ Centre for Green Chemistry, P.O. Box 23, Monash University, Melbourne 3800, \\ Victoria, Australia
}

\begin{abstract}
Teaching green chemistry at Monash University started in earnest in 1999 with the launch of a 12-lecture final year undergraduate course. The course was designed to introduce students to the field of green chemistry. The students had no prior knowledge of the field or indeed what green chemistry meant. The course covered a definition of green chemistry, historical issues, and challenges, together with an introduction to the principles and tools of green chemistry, evaluating the effects of chemistry and technology, general areas of green chemistry, and presenting examples of successful and developing green chemistry technologies. Several general references were used, although specific examples were from the primary literature. Another feature of the course was the analysis of recent papers. In addition, experiments linking into the course have been developed, and there are plans for postgraduate training, professional development, and community outreach involving postgraduate candidates.
\end{abstract}

\section{OVERVIEW}

Green chemistry is a major research activity at Monash University, mainly in the School of Chemistry, and also with various contiguous divisions of the Commonwealth Scientific \& Industrial Research Organisation (CSIRO). Initially, it was supported as a special research initiative by Monash University (1999), then by the Australian Research Council as a Special Research Centre, with significant funding possible for up to nine years. Interest in teaching green chemistry has been in parallel with the research initiatives, and the first module developed was delivered July-October 1999. In the first two years, this comprised 12 lectures and 2 tutorials with set problems, including the analysis of recent papers. Then, in 2001, experiments were developed linking in with this module, and two postgraduate Master's degree modules are under development.

In the Australian context, the undergraduate academic year commences in late February/early March, and finishes late October/early November, and a level-three module is at the final year level of a three-year B.Sc. degree. After the B.Sc., there is a one-year Honors degree which has a large research component, and this is the standard entry into the Ph.D. program. Another entry to the Ph.D. program is through a Master's degree, either from the Honors degree or the B.Sc. In addition to the third-level course, there is also an optional short research project in green chemistry, and it is noteworthy that there is continued interest in Honors research projects in green chemistry, and also for the Master's and Ph.D. degrees. Recently, approximately half the Honors students have elected projects in green chemistry, and there are now over $20 \mathrm{Ph} . \mathrm{D}$. candidates undertaking projects in the field. As part of the Ph.D. training there are planned workshops and a community outreach program where candidates visit secondary schools spreading the message about green chemistry. In moving to the University of Leeds during

\footnotetext{
*Lecture presented at the IUPAC CHEMRAWN XIV Conference on Green Chemistry: Toward Environmentally Benign Processes and Products, Boulder, Colorado, USA, 9-13 June 2001. Other presentations are published in this issue, pp. 1229-1330.

${ }^{\dagger}$ Corresponding author: present address: School of Chemistry, University of Leeds, Leeds LS2 9JT UK
} 
2001, Prof. Raston has been given approval to present a 20-lecture level-three course there commencing in early 2002.

A high success of the third-year-level course is suggested by the high number of students enrolled in it as an elective course-17 in 1999, and surprisingly with an additional 8 students attending the course but not enrolled, and staying on for all lectures and tutorials! The high average mark, with no failures, and subsequent interest by the students in research degree projects in green chemistry, is also noteworthy.

\section{THIRD-YEAR-LEVEL COURSE SYNOPSIS/AIMS}

The aims of the third-year-level course are as follows:

- to define green chemistry and give an overview of the historical developments leading to the emergence of green chemistry, and the associated challenges

- $\quad$ to introduce the principles of green chemistry and establish the arguments for our need to recognise green chemistry criteria in the practice of chemistry-evaluating the effects of chemistry and technology, and how the 2020 Vision-U.S. Chemical Industry document essentially embraces the principles of green chemistry.

- to develop the tools and general areas of green chemistry

- to present examples of successful and developing green chemistry technologies including: (i) alternative feedstocks and starting materials; (ii) clean oxidation technologies; (iii) the utility of solvents in green chemistry, alternative reaction conditions, e.g., supercritical $\mathrm{CO}_{2}$ as a substitute medium for chemical synthesis and catalysis, water (in association with microwave technology), and ionic liquids as media for chemical reactions; (iv) processing using carbon dioxide-supercritical carbon dioxide-assisted aerolization for thin-film deposition, fine powder generation, and drug delivery; (v) pollution prevention via molecular recognition and self assembly; and (vi) biocatalysis

- $\quad$ to be familiar with recent trends in green chemistry in the widest context

- to be able to perform critical analysis of recent short papers in the literature- "greenness"

\section{REFERENCE MATERIAL}

Several general references [1-5] were used, but for the above examples most of the material was from the original journal articles, for example, refs. 6 to 14, and a feature of the course was the analysis of recent short papers in the field, from leading journals, including the Journal of the American Chemical Society, Chemical Letters, Angewandte Chemie, Chemical Communications, and Green Chemistry. The inclusion of recent, cutting-edge research papers allows for continual updating of the course so that the content remains current and reflects recent advances in green chemistry research and technology development.

\section{ASSESSMENT}

In addition to the lecture course, there were two tutorial classes with set problems for the students to work through prior to the tutorial, and to actively participate in the tutorial. Some typical questions are given below. In 1999-2000, the examination consisted of 105-minute papers with questions on definitions and problems, and the analysis of a short paper, all of the type covered in tutorials. In 2001, with the amalgamation of the green chemistry course and a course on energy chemistry to form a new 24-lecture, 12-tutorial course called sustainable chemistry, the assessment has changed to a 90-minute green chemistry and 60-minute energy chemistry paper. There is also an assessed written assignment and practical component. 
1. Adipic acid is a key intermediate in the production of nylon 66. Discuss the current method of production of adipic acid and its associated problems, and how this can be largely overcome using the Noroyi method, starting from cyclohexene [Science, 281, 1646 (1998)].

2. Discuss the green chemistry approaches to the synthesis of caprolactam, the precursor for the synthesis of nylon 6 .

3. (a) What are the advantages and disadvantages of carrying out organic reaction in water?

(b) Discuss the hydrophobic effect of water in the context of Diels Alder reactions [see R. Breslow, Acc. Chem. Res. 24, 159 (1991)].

4. Critically assess the chemistry in the following paper in relation to it fulfilling the principles of green chemistry: "A Convenient Synthesis of Alkyl Substituted $p$-Benzoquinones from Phenols and $\mathrm{H}_{2} \mathrm{O}_{2}$ over TiAPO-5 Molecular Sieve Catalysts", R. J. Mahalingam and P. Selvam, Chem. Lett. 455 (1999).

\section{EXPERIMENTS}

A policy of the School of Chemistry at Monash is the integration of laboratory classes with lecture work. To this end, some experiments have been developed. The first of these is an environmentally benign synthesis of adipic acid starting from 2-cyclohexene, eq. 1 [7]. No solvent is used with product crystallizing from the aqueous phase. Outcomes of the experiment cover learning techniques and compound characterization, identification of a by-product, and greater appreciation of the principles of green chemistry.
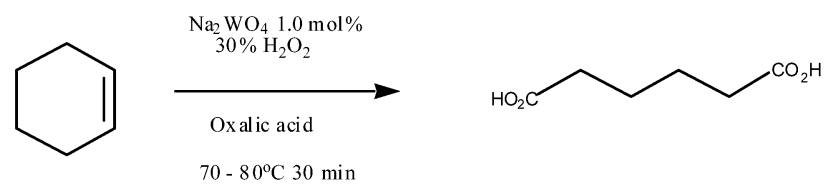

Another experiment deals with two benign syntheses of 3-carboxycoumarins, solventless and an aqueous slurry, Scheme 1 [7], the learning outcomes being similar to those in the above experiment.

Scheme 1

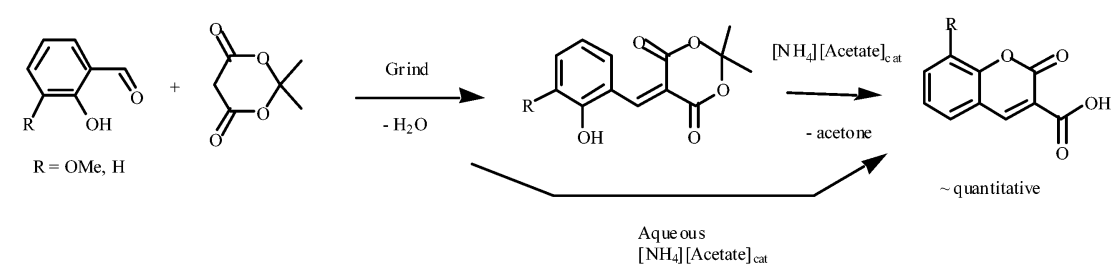

\section{CONCLUSIONS}

The final lecture touched on future trends in green chemistry, which will see major advances in the above areas. These include the use of so called combinatorial chemistry (rapid throughput screening) to find lead compounds of importance in the pharmaceutical industry, catalysis and materials science, biomimetic reactions, multifunctional reagents, chemistry which prevents problems and solves current problems (e.g., $\mathrm{CO}_{2}$ as a feedstock), biomass conversion, energy considerations, analytical methodology, noncovalent derivatization, nanotechnology, and benign mineral processing, particularly for local unique ore bodies in the Australian context.

Green chemistry is now an important aspect of teaching chemistry at Monash University, at the undergradaute level and into postgraduate activities. The same is also becoming a reality at the 
University of Leeds, with a 20-lecture module starting in 2002. It is noteworthy there is a well-established research ethos in green chemistry at Leeds, largely through the efforts of Chris Rayner and Tony Clifford on supercritcial $\mathrm{CO}_{2}$ as a reaction medium, and in separation technology.

\section{ACKNOWLEDGMENTS}

The authors would like to acknowledge the assistance from Terry Collins during 1998 in getting started on teaching green chemistry, the assistance of Chris Strauss in teaching aspects of water/microwave chemistry, David Brennan in presenting a lecture on life cycle assessment, and Brindaban Ranu for a guest lecture on supported reagents.

\section{REFERENCES}

1. P. T. Anastas and J. C. Warner. Green Chemistry: Theory and Practice, Oxford Science Publications, New York (1998).

2. P. Anastas and T. Williamson. Green Chemistry: Frontiers in Benign Chemical Synthesis and Processes, Oxford Science Publications, New York (1998).

3. J. H. Clark. Green Chem. 1, 1 (1999); J. H. Clark. Chem. Britain 43, October (1998).

4. R. G. Sheldron. Chem. Ind. 903 (1992).

5. 2020 Vision - U.S. Chemical Industry (1996).

6. K. Sato, M. Aoki, R. Noyori. Science. 281, 1646 (1998); Y. Deng, Z. Ma, J. Chen. Green Chem. 1, 275 (1999).

7. C. L. Raston and J. Scott. Green Chem. 2, 49 (2000); J. L. Scott and C. L. Raston. Green Chem. 2, 245 (2000).

8. C. R. Strauss. Aust. J. Chem. 52, 83 (1999).

9. K. M. Draths and J. W. Frost. J. Am. Chem. Soc. 116, 399 (1994).

10. C.-H. Li and W.-C. Zhang. J. Am. Chem. Soc. 120, 9102 (1998).

11. C. Li and T. Chen. Organic Reactions in Aqueous Media, Wiley Interscience, New York (1997).

12. R. S. Oakes, T. J. Heppenstall, N. Shezad, A. A. Clifford, C. M. Rayner. Chem. Commun. 1459 (1999).

13. J. Holbrey and K. Seddon. Dalton Trans. 2133 (1999).

14. D. MacFarlane, P. Meakin, J. Sun, N. Amini, M. Forsyth. J. Phys. Chem. B. 103, 4164 (1999). 